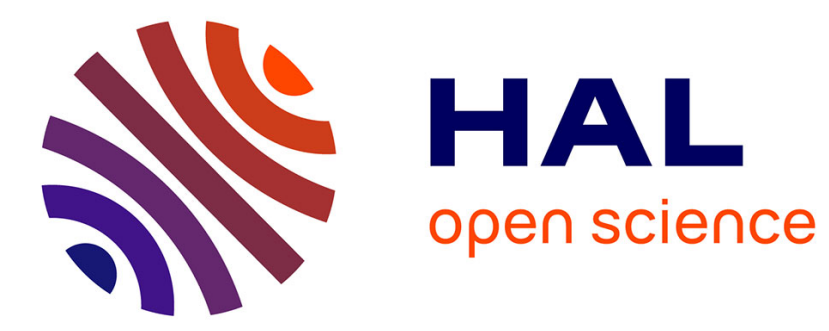

\title{
ÉTUDE DE LA DÉCROISSANCE DE 158Tm
}

\author{
P. Aguer, C. Liang, J. Libert, P. Paris, A. Peghaire, A. Charvet, R. Duffait, \\ G. Marguier
}

\section{To cite this version:}

P. Aguer, C. Liang, J. Libert, P. Paris, A. Peghaire, et al.. ÉTUDE DE LA DÉCROISSANCE DE 158Tm. Colloquium on Nuclear Surface, 1975, Dijon, France. pp.C5-104-C5-104, 10.1051/jphyscol:1975521 . jpa-00216376

\section{HAL Id: jpa-00216376 https://hal.science/jpa-00216376}

Submitted on 1 Jan 1975

HAL is a multi-disciplinary open access archive for the deposit and dissemination of scientific research documents, whether they are published or not. The documents may come from teaching and research institutions in France or abroad, or from public or private research centers.
L'archive ouverte pluridisciplinaire HAL, est destinée au dépôt et à la diffusion de documents scientifiques de niveau recherche, publiés ou non, émanant des établissements d'enseignement et de recherche français ou étrangers, des laboratoires publics ou privés. 


\title{
ÉTUDE DE LA DÉCROISSANCE DE ${ }^{158}$ Tm
}

\author{
P. AGUER, C. F. LIANG, J. LIBERT, P. PARIS, A. PEGHAIRE, \\ A. CHARVET $(*)$, R. DUFFAIT $\left({ }^{*}\right)$ et G. MARGUIER $\left(^{*}\right)$ \\ C.S.N.S.M., Orsay et I.P.N. $\left({ }^{*}\right)$, Lyon, France
}

\begin{abstract}
Résumé. - Les niveaux excités de ${ }^{158} \mathrm{Er}$ ont été étudiés par la décroissance $\left(\mathrm{CE}, \beta^{+}\right)$de ${ }^{158} \mathrm{Tm}$ $\left(T_{1 / 2}=3,9 \mathrm{~min}\right)$. Les sources étaient obtenues par réaction, $(\mathrm{p}, x n)$ sur une cible d'erbium naturel, suivie d'une séparation en ligne avec le séparateur Isocèle d'Orsay. Les rayonnements $\gamma$ ont été étudiés par des méthodes classiques de coïncidences bi-dimensionnelles $2048 \times 2048$ canaux, les électrons de conversion ont été observés à l'aide d'un détecteur Si(Li) placé dans un sélecteur magnétique.

Cent quatre-vingts rayonnements $\gamma$ de $100 \mathrm{keV}$ à $3 \mathrm{MeV}$ ont été attribués à la désexcitation de ${ }^{158} \mathrm{Tm}$, et cent vingt d'entre eux ont été placés sur un schéma de niveaux. Une interprétation de ce schéma est proposée dans le cadre d'un modèle de noyaux déformés. C'est ainsi que, outre la bande de rotation bâtie sur le niveau fondamental, les bandes de rotation suivantes sont proposées :
\end{abstract}

\begin{tabular}{ccr}
$K \pi$ & $I$ & \multicolumn{1}{c}{$E_{\gamma}$} \\
$-0^{+}$ & $0^{+} \beta$ & - \\
& $2^{+} \beta$ & 806,4 \\
$2^{+}$ & $4^{+} \beta$ & 989,0 \\
& $2^{+} \gamma$ & 1257,2 \\
$0^{-}$ & $3^{+} \gamma$ & 820,1 \\
& $3^{-}$ & 1043,4 \\
& $1^{-}$ & 1342,0 \\
& & 1418,3
\end{tabular}

L'examen des $\log f_{\mathfrak{t}}$ nous fait enfin proposer pour le niveau fondamental de ${ }^{158} \mathrm{Tm}$ les spin et parité $2^{-}$.

Abstract. - The decay of $4 \mathrm{~min}{ }^{158} \mathrm{Tm}$ has been investigated with on line mass-separated samples obtained from the Orsay Isocele separator. Measurements of gamma rays, conversion electron lines and gamma-gamma bi-dimensional coincidences were performed. About 180 transitions were ascribed to the decay and two thirds of them were placed in a decay scheme. The beta band and the gamma band were identified with bandheads situated at 806.40 and $820.13 \mathrm{keV}$ respectively. In addition, a number of other vibrational bands (beta-gamma, beta-beta, $K^{\pi}=0^{-}$and $1^{-}$) are proposed. The decay properties of those bands are discussed in the framework of current nuclear models. The $\log \mathrm{ft}$ values suggest a $2^{-}$assignement for ${ }^{158} \mathrm{Tm}$ with the possible configuration (p $404 \downarrow-\mathrm{n} 521 \uparrow$ ).

(**) Article publié dans Nuclear Physics A 249 (1975) 239. 